\title{
THE INFLUENCE OF SPIN REORIENTATION TRANSITION ON ELECTRICAL PROPERTIES OF SOME W-TYPE HEXAGONAL FERRITES
}

\section{P.VENUGOPAL REDDY}

Department of Physics, Osmania University, Hyderabad-500 007, INDIA.

Abstract - In contrast to the general belief that Spin Reorientation Transitions (SRT) influence to large extent magnetic properties of some cobalt containing ferrites materials, it was observed by the author for the first time that the SRTs also affect the electrical transport properties such as thermopower (S), electrical conductivity $(\sigma)$ and dielectric constant $\left(\varepsilon^{\prime}\right)$, of some hexagonal W-type ferrites. More surprisingly, it was also observed that SRTs are found to influence neither magnetic properties nor electrical properties in the case of SrZnCo-W type ferrites. A qualitative model to explain the observed behaviour is proposed.

\section{INTRODUCTION}

It is well known that the hexagonal ferrites with W-type structure exhibit uniaxial anisotropy along $\mathrm{C}$-axis and that when $\mathrm{Co}^{2+}$ is added even in small proportions are expected to show various types of magnetic anisotropy, characterised by an easy axis, or an easy plane or an easy cone of magnetisation. In fact number of investigators [1-5] studied the phenomenon of "Influence of cobalt doping on number of physical properties of these ferrites" and concluded that the orientation of magnetic ions changes by varying cobalt concentration in the case of $\mathrm{BaZn}_{2-x} \mathrm{Co}_{\mathrm{x}} \mathrm{Fe}_{16} \mathrm{O}_{27}$. It was also reported that when 1.0 mole of cobalt is added, the spin direction is found to change by $180^{\circ}$ [6-8]. This change of spin direction hereafter known as the spin reorientation transition (SRT) is expected to influence number of physical properties - the influence being more prominent in the case of magnetic properties. With a view to understand the influence of the so called SRTs on electrical properties such as electrical conductivity, thermopower and dielectric properties, number of W-type ferrite systems were chosen and the results of such a study are presented here.

\section{EXPERIMENTAL}

After characterising the samples by undertaking $\mathrm{XRD}$, bulk density, porosity, etc., measurements, thermopower, electrical conductivity, dielectric constants, etc., studies were also carried out by different techniques, mainly to study the influence of SRTs on these non-magnetic properties. Details of the experimental techniques for the measurement of these parameters are given elsewhere [9-11].

\section{Results AND Discussion}

The variation of Seebeck coefficient, electrical conductivity and dielectric constant as a function of composition and temperature for all the samples is shown in Figs. 1 \& 2. It is evident from Figure 1 that all the measured electrical parameters are found to exhibit anomalous behaviour in the vicinity of 1.0 mole concentration of cobalt. It is clear from Figure 2 that, as the temperature increases, all the three electrical parameters are found to exhibit yet another transition at a temperature $\left(\mathrm{T}_{1}\right)$ much below their respective Curie temperatures $\left(\mathrm{T}_{d}\right)$. In contrast to this, sample without cobalt concentration is not found to exhibit any type of change of slope or transition at the so called transition temperature $\left(T_{1}\right)$.

With a view to understand the reasons for the occurrence of a transition at $T_{1}$, a thorough survey of literature was undertaken and it was observed that there are such transitions especially in the temperature region from $430-530 \mathrm{~K}$ in the case of certain hexagonal ferrites. For instance, in the case of $\mathrm{BaCo}_{1.8} \mathrm{Fe}_{0.2} \mathrm{Fe}_{16} \mathrm{O}_{27}$, a change of slope in the 
magnetisation versus temperature plot was reported at $445 \mathrm{~K} \mathrm{[12].} \mathrm{The} \mathrm{authors} \mathrm{attributed} \mathrm{the} \mathrm{kink} \mathrm{to}$ the spin reorientation wherein the direction of the magnetisation changed from planner to an easy axis. Further, while studying the temperature variation of thermal expansion coefficient $(\alpha)$ a sudden drop in the case of $\mathrm{Ni}_{2-y} \mathrm{Co}_{y}-\mathrm{W}$ hexagonal ferrite at 500 $\mathrm{K}$ was also reported [13]. In the present investigation also the plots of all the three electrical parameters versus temperature are found to exhibit a change of slope in the same temperature region i.e., between 460 to $480 \mathrm{~K}$. A comparison of all the transitions reported along with those of the present investigation indicates that the temperature at which the spin reorientation takes place may vary from sample to sample and that it may depend on the cobalt concentration, the quenching and sintering temperature, and many other preparatory conditions. However, the temperature region where the transition is likely to occur lies between 450 and $500 \mathrm{~K}$. As such, the transition observed at $T_{1}$ may be attributed to the reorientation of the spins. The absence of such reorientation transition for the first composition is logical due to the fact that it does not contain cobalt. With a view to throw some more light on the subject and also to generalise the results, saturation magnetisation $\left(\sigma_{s}\right)$ measurements of cobalt doped $\mathrm{Sr}-\mathrm{Zn} \mathrm{W}$-type ferrites have also been undertaken over a temperature range $300-800 \mathrm{~K}$ by using a Vibrating Sample Magnetometer. It is surprising to note that the generally observed SRTs in $\sigma_{s}$ vs cobalt concentration plots and in $\sigma_{\mathrm{s}}$ vs temperature behaviour are found to be totally absent. In order to understand the unexpected behaviour, a thorough examination of the entire concept of the phenomenon of SRTs have been made. It has been speculated that $\mathrm{Sr}$ ion which generally has the tendency to supress the functioning of a dopant ion might be responsible for the absence of SRTs in cobalt doped $\mathrm{Sr}-\mathrm{Zn} \mathrm{W}$-Type ferrite materials. In fact, the suppression of high profile ferro-electric behaviour of $\mathrm{BaTiO}_{3}$ to a large extent when $\mathrm{Ba}$ is replaced with $\mathrm{Sr}$, might corroborate the above arguement. More recently another interesting result has been observed. It was found that when $\mathrm{Ba}-\mathrm{Zn}$ $\mathrm{Co}-\mathrm{W}$ ferrites are quenched in liquid nitrogen instead of air, the so called SRTs have become more prominent and clearer. Further studies are in progress to understand all these peculiar type of behaviours.

\section{REFERENCES}

[1] F.K.Lotgering, U.Enz and J.Smit, Phil.Res.Rep., vol.16, pp.441, 1961.

[2] T.M.Perikalina and A.V.Zalesskii, Sov.PhysJETP, vol.19, pp.1337, 1964.

[3] I.I.Yamzin, R.A.Sizov, S.Zheludev, T.M.Perikalina and A.V.Zalesskii, Sov.PhysJETP, vol.23, pp.395, 1968.

[4] E.M.Smirnovskaya, T.M.Perikalina and S.A. Cherkezyan, Sov.Phys.Solid State, vol.19, pp.1572, 1977.

[5] V.F.Belov, T.A.Khimich, N.M.Shipku, T.S.Zheludev, E.V.Kornevr and N.S.Ovanesyan, Sov.Phys-JETP, vol.37, pp.1089, 1973.

[6] G.Albanese, E.Calabrase, A.Siru and F.Licci, Hyperfine Interactions, vol.28, pp.487, 1986.

[7] A.Paoluzi, F.Licci, O.Moze, G.Turilli, A.Deriu, G.Albanese and E.Calabrase, J.Appl.Phys., vol.63, pp.5074, 1988.

[8] E.P.Naiden, V.I.Maltsev and G.I.Ryabtse, Phys.Stat.Sol.(a), vol.120, pp.209, 1990.

[9] V.D.Reddy and P.V.Reddy, Phys.Stat.Sol.(a), vol.142, pp.451, 1994.

[10] P.V.Reddy, T.S.Rao and S.M.D.Rao, J.Less Common Met., vol.79, pp.1, 1989.

[11] V.D.Reddy, Y.Purushotham, M.B.Reddy and P.V.Reddy, Mod.Phys.Lett.B, vol.29, pp.1461, 1996.

[12] S.P.Kuntsevich and V.P.Palekhin, Sov.Phys.Solid State, vol.20, pp.1661, 1978.

[13] V.N.Gorbach and Yu.A.Mamulai, Sov.Phys.Solid State, vol.17, 1975. 


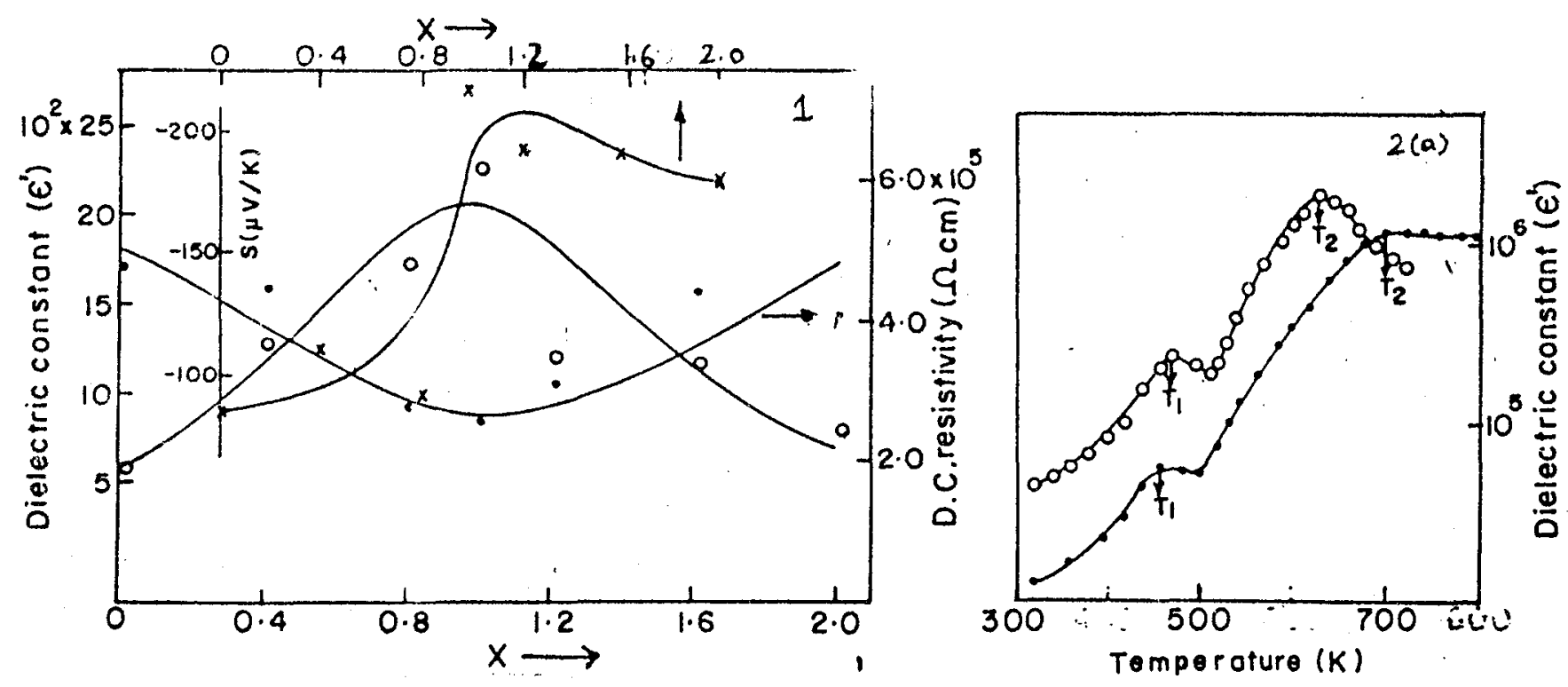

fig.1. Eloctrlcel paramotors vorsus sobalt coritart $(x)$
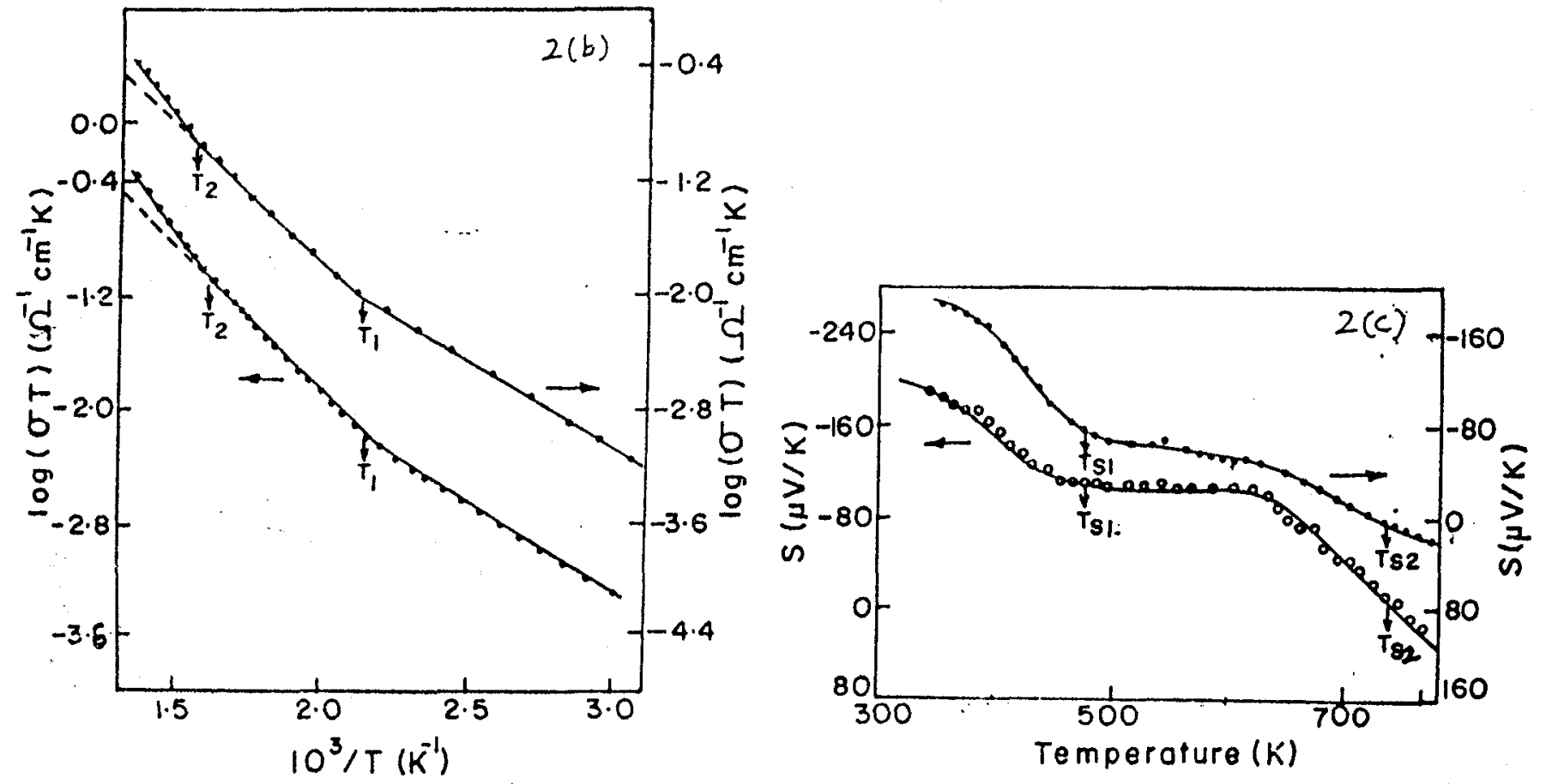

Fig.z. Varlation of (a) delectric constant, (b) electrical corductivity, $i$ ) Serbeck coefflcient with temperature 